\title{
Dynamic Scale-Space Theories
}

\author{
Alfons H. Salden ${ }^{\star}$ \\ INRIA Sophia Antipolis, 2004 route des Lucioles, BP 93, \\ F-06902 Sophia Antipolis Cedex, France
}

\begin{abstract}
In this paper image formation is quantised by imposing an image induced connection and computing the associated torsion and curvature. Next dynamic scale-space theories are proposed that couple to and smooth the image formation itself.
\end{abstract}

\section{Introduction}

A major reason for developing scale-space theories [1] is to find a stable and reproducible image by sieving noise, by detection, conservation, restoration and/or enhancement of topologically interesting objects like edges, corners, and more global entities like volumes, and by data compression and reduction. A requirement imposed on all these theories is that the so-called scale-space operator causes a smoothing of the image that is invariant or slightly affected by certain sets of transformations such as the group of anamorphoses and the group of diffeomorphisms of the image, and transformations caused by noise. Subsequently the image can initiate and control the dynamics and architecture of autonomous artificial systems like robots that, of course, influences the image formation itself. In these contexts we'll restrict ourselves to presenting so-called dynamic scale-space theories [1], in which dynamic refers to the coupling of the paradigm to the image formation process.

Normally one assumes in the scale-space theories that the connection on the image domain is flat. This implies that the image domain or the objects defined on them are neither curved nor twisted in a modern geometric sense. For example, in linear scale-space theory one takes as the base-manifold just Euclidean space. Another example is shortening flow theory for curves and surfaces solved by means of the level set method. Here one encounters notions like curvature and torsion coinciding with a classical geometry living on e.g. the isophotes and flowlines, but only in the context of zero-forms being invariant under classical transformation groups and being factors in the connection coefficients of a flat connection on these objects. The resulting smoothing schemes lead to a family of images in which non-isolated singularity and discontinuity sets in the grey-valued image are destroyed instantaneously.

* This work was supported by the Netherlands Organisation of Scientific Research, grant nr. 910-408-09-1, and by the European Communities, H.C.M. grant nr. ERBCHBGCT940511. 
Simultaneously anisotropic scale-space theories were developed to retain discontinuity sets of the grey-valued image as much as possible [2]. The latter theories found a solid foundation in [1] where it was pointed out for the first time that Alvarez and Weickert used without realising it modern geometry. Measuring the homogeneity of the image gradient field to steer the diffusion process on the grey-valued image is equally well expressed as measuring the inhomogeneity of the image gradient field to control it.

Introducing an image induced connection [1] characterised by a non-zero torsion and curvature the image formation can be captured in terms of so-called translation and/or rotation vector density fields. Such vector fields one identifies in defect theory and gauge field theories with a so-called Burgers and Frank vector fields, respectively. These vector fields are in particular non-zero at so-called defect or cut lines where the physical properties of media are multi-valued. The reason for these properties being multi-valued lie in the fact that actually different media touch each other or that material has been inserted or removed causing disclinations and dislocations of e.g. the crystal lattice. Such lines happen to be also retrievable in images by the same geometric expertise. They are normally called ridges and ruts in cartography. Having quantised the inhomogeneity in image formation in terms of vector density fields the relaxation of the image or its formation coupled to those fields comes into sight.

The organisation of this paper is as follows. In section 2 we will very briefly treat modern geometry and illustrate its use in quantising the image formation at ridges, ruts and other type of topological objects. Finally, in section 3 the image induced connections are used to formulate dynamic scale-space theories.

\section{Modern Geometry of Image Formation}

As in the sequel the presentation of the dynamic scale-space theories heavily relies on modern geometry its most important ingredients are summarised. In subsection 2.1 differential geometry and in subsection 2.2 integral geometry are briefly treated. For a more thorough treatment of modern geometry the reader is referred to [1] and the references therein. In subsection 2.3 modern geometry is applied to the problem of finding the essential physical objects in images.

\subsection{Differential Geometry}

Let $M$ be a $D$-dimensional image domain parametrised by canonical coordinates $p=\left(p^{1}, \ldots p^{D}\right)$. Now consider the frame bundle $F \equiv P(M, \pi, A(D, \mathbb{R}))$ where $P$ is the total space consisting of all frames $\Phi_{p}$ at each point $p \in M, \pi: P \rightarrow M$ is the projection and $A(D, \mathbb{R})=G L(D, \mathbb{R}) \triangleright T(D, \mathbb{R})$ the full affine group, where $G l(D, \mathbb{R})$ is the general linear group and $T(D, \mathbb{R})$ the translational group. In this context let's define a local frame as follows.

Definition 1. A local frame $\Phi_{p}$ is defined by:

$$
\Phi_{p}=\left(x ; e_{1}, \ldots, e_{D}\right)(p)
$$


where the vectors $\left(x, e_{1}, \ldots e_{D}\right)(p)$ span the local tangent space $T_{p} A(D, \mathbb{R})$.

Now an affine connection $\Gamma$ in the frame bundle $F$ is defined as follows.

Definition 2. An affine connection $\Gamma$ in the frame bundle $F$ is defined in terms of the Lie algebra $\mathcal{G}(D, \mathbb{R})$-valued connection one-forms $\left(\omega^{i}, \omega_{i}^{j}\right)$ and the frame vectors $\left(x, e_{1}, \ldots e_{D}\right)$ through the following equality:

$$
\nabla x=\omega^{i} e_{i}, \nabla e_{i}=\omega_{i}^{j} e_{j},
$$

where $\nabla$ is the covariant differential operator.

The affine connection $\Gamma$ satisfies so-called structure equations:

Theorem 3. Given an affine connection $\Gamma$ in the frame bundle $F$, defined in (2), then the connection one-forms satisfy the following structure equations:

$$
D \omega^{i}=d \omega^{i}+\omega_{k}^{i} \wedge \omega^{k}=\Omega^{i}, D \omega_{j}^{i}=d \omega_{j}^{i}+\omega_{k}^{i} \wedge \omega_{j}^{k}=\Omega_{j}^{i},
$$

where $d$ the ordinary exterior derivative, $\wedge$ is the wedge product, $D$ the covariant derivative, $\Omega^{i}$ is the torsion 2-form and $\Omega_{j}^{i}$ is the curvature 2-form.

In turn the torsion and the curvature 2-form satisfy so-called Bianchi identities:

Theorem 4. Let $\Gamma$ be an affine connection in the frame bundle $F$ with torsion 2 -form $\Omega_{0}^{i}$ and curvature 2 -form $\Omega_{j}^{i}$. The integrability conditions for the structure equations, that are the Bianchi identities, are given by:

$$
D \Omega^{i}=\Omega_{j}^{i} \wedge \omega^{j}, D \Omega_{j}^{i}=0,
$$

\subsection{Integral Geometry}

Following Cartan [1] one can apply a displacement to determine the translation vector field and the rotation vector fields to operationalise the torsion and the curvature of the frame bundle $F$ with connection $\Gamma$.

Definition 5. Let $\Gamma$ be a connection in the frame bundle $F$. The translation vector field $b$ and the rotation vector fields $f_{i}$ determined by the connection are defined by:

$$
b=\oint_{C} \nabla x, f_{i}=\oint_{C} \nabla e_{i},
$$

where $C$ is an infinitesimally small closed loop and boundary of a 2-dimensional submanifold $S$ of $M$ with the same induced connection $\Gamma$. The sense of traversing the loop is chosen such that the enclosed submanifold is to the left.

On the basis of the connection one forms $\omega_{0}^{i}$ a foliation of the manifold $(M, \Gamma)$ can be realised and choosing $\frac{1}{2} D(D-1)$ pairs of them will yield submanifolds containing the desired submanifold $S$. These integral invariants are intrinsic 
vectors of the submanifold $(S, \Gamma)$ and also of the manifold $(M, \Gamma)$. Using Stokes' theorem the translation and rotation vector fields can be expressed as [1]:

$$
b=\int_{S} \Omega^{i} e_{i}, f_{\imath}=\int_{S} \Omega_{i}^{j} e_{j} .
$$

At branching points the translation and rotation vector fields satisfy the following superposition principles (conservation laws for "topological" currents):

$$
B=\sum b, F_{i}=\sum f_{i} .
$$

The latter principles can be conceived as the integral geometric formulations of the Bianchi identities in the previous subsection.

\subsection{Application}

Let's find the essential physical objects of an image that are invariant under the group of (not necessarily total grey-value preserving) diffeomorphisms of the image caused by active transformations of the scene. The latter active transformations may lead to e.g. anamorphoses of the image or integrable deformations of the net of flowlines and isophotes. It's clear that the set of (non)-isolated singularities and the set of discontinuities of the image remain the same topologically equivalent sets under these transformations. The vanishing of the image gradient is not affected, neither are discontinuities. A set of nonisolated singularities occurs, for example, for images like $L_{0}^{n}(x, y)=-\Re\left((x+i y)^{\frac{3 n+1}{2}}\right), n \in \mathbb{N}$. It is not so obvious that this invariance also holds for the landscape of ridges and ruts of a smooth image [1]. The latter topological equivalence of non-isolated singularity sets, ridges and ruts can be explained by the fact that across them either the flowlines or the isophotes have opposite convexity. In order to detect subsesequently e.g. ridges and ruts in a two-dimensional grey-valued image a non-local integral geometric difference operation is needed along isohotes with respect to the normalised curvature vector field. At ridges and ruts application of this curvature operator then yields a vectorial Dirac pulse.

Now let us demonstrate that an integral geometric operation suffices to detect certain types of singularity sets. In figure 1 the length of the translation vector field $b$ for a discretised input image $L_{0}$ on a two-dimensional Euclidean space $E^{2}$ is computed by means of linear scale-space theory [1]. The set of nonisolated singularities will instantaneously disappear upon linear scaling, but the "apparent ridge" and endpoint occurring normally in e.g. fingerprint images can be nicely detected. Note that here we only imposed invariance under the group of Euclidean movements allowing us to consider e.g. the Euclidean differential geometry of the net of isophotes and flowlines of the smoothed images. This geometric and topological expertise enables us to come up with the necessary instructions for the construction of an image.

The proposed non-local topological and integral geometric analysis can be extended to grey-valued images defined on higher dimensional image domains. 
For example in the case of three-dimensional grey-valued images normally the unit normal frame field to an isophote will be unique but for some singular ones they will be multi-valued. Again topological operations and slot-machines for reading out torsion or curvature can be used to locate singularity or discontinuity sets of non-constant co-dimension.
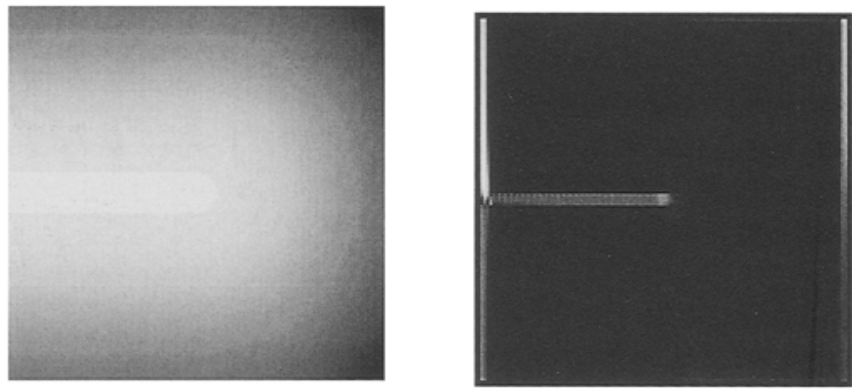

Fig. 1. Left frame: a $256 \times 256$ pixel-resolution discrete input image $L_{0}(x, y)=L_{0}^{0}(x, y)$. Right frame: the Euclidean length of the translation vector $|b|$ for a linearly scaled version of that image.

\section{Dynamic Scale-Space Theories}

In subsection 3.1 dynamic scale-space theories for smoothing the input image are derived by determining the Beltrami-Laplace operator consistent with a particular choice of an image induced connection and metric. In subsection 3.2 dynamic scale-space theories for smoothing the image formation are derived by coupling the smoothing of the image formation to itself.

\subsection{Input Images}

First let us denote with label $r e f$ and $i n d$ aspects related to the geometry of the image domain (the camera system) and the induced geometry by the input image, respectively. Assume that dynamic scale-space theories for the input image can be based on the following conservation law on a region $\mu_{i n d}$ of the image domain $M_{i n d}$ with boundary $\partial \mu_{i n d}$ :

$$
\int_{\mu_{i n d}} \frac{\partial L}{\partial s} \eta\left(\mu_{i n d}\right)=\int_{\partial \mu_{i n d}} \gamma_{i n d}\left(\nabla_{i n d} L, \partial^{n} \mu_{i n d}\right) \eta\left(\partial \mu_{i n d}\right)
$$

where $\partial^{n} \mu_{\text {ind }}$ is a normal field, $\nabla_{\text {ind }}$ is the induced covariant derivative constructed on the basis of the induced connection $\omega_{i n d}, \gamma_{i n d}$ is an induced metric tensor, $\eta\left(\mu_{i n d}\right)$ and $\eta\left(\partial \mu_{i n d}\right)$ are volume measures on the interior $\mu_{i n d}^{0}$ and the boundary $\partial \mu_{i n d}$, respectively. Nothe that the metrics need not to be compatible 
with the connections [1]. Using the divergence theorem and reflective boundary conditions these dynamic scale-spaces are governed by the following Cauchy problem:

$$
\begin{aligned}
\frac{\partial L}{\partial s} & =\gamma_{i n d}^{\alpha \beta} \nabla_{i n d, \alpha} \nabla_{i n d, \beta} L \\
\frac{\partial L}{\partial n_{\text {ind }}} & =0 \text { on } \partial \mu_{\text {ind }} \times \mathcal{R}_{s}, n_{\text {ind }} \in \partial^{n} \mu_{\imath n d}, \\
L(x, 0) & =L_{0}(x)
\end{aligned}
$$

in which the first equation represents the scaling operation, the second equation a reflective boundary condition ensuring the conservation of total flux and the third equation states the initial condition.

Requiring the dynamic scale-spaces for the input image to be invariant under spatially homogeneous grey-value transformations and applying a similar conservation principle as above for geometries induced by the input image, the divergence theorem and a variational principle the dynamic scale-spaces can be shown to be governed by the following Cauchy problem [1]:

$$
\begin{aligned}
\frac{\partial L}{\partial s} & =-\gamma_{r e f}^{\alpha \beta} \nabla_{r e f, \alpha} L \triangle_{i n d} x_{\beta}, \\
\frac{\partial L}{\partial n_{\text {ind }}} & =0 \text { on } \partial \mu_{i n d} \times \mathcal{R}_{s}, n_{\text {ind }} \in \partial^{n} \mu_{i n d}, \\
L(x, 0) & =L_{0}(x),
\end{aligned}
$$

with $\triangle_{\text {ind }}$ the induced Beltrami-Laplace operator given by:

$$
\triangle_{i n d}=-\gamma_{i n d}^{\alpha \beta} \nabla_{2 n d, \alpha} \nabla_{i n d, \beta} .
$$

Example 6. The smoothing of a grey-valued input image on two-dimensional Euclidean space $E^{2}$ is normally steered by the image gradient through the conductivity tensor [2]. Alternatively, one could steer the heat capacity on the basis of the image gradient. Choosing a flat connection and a metric equal to

$$
\gamma=\exp \left(-\frac{L_{i} L_{i}}{\lambda^{2}}\right) d x^{p} \otimes d x^{p},
$$

one obtains, upon substitution into equation (1) and (3), and assuming the image domain not to be bounded, as Cauchy problem for the input image:

$$
\frac{\partial L}{\partial s}=\exp \left(-\frac{L_{i} L_{i}}{\lambda^{2}}\right) \triangle_{r e f} L, L(\cdot, 0)=L_{0}(\cdot),
$$

where $\lambda$ a contrast parameter.

Example 7. Instead of steering the smoothing of a grey-valued input image on two-dimensional Euclidean space directly as in the above example one can also 
prefer to control the smoothing indirectly choosing the connection and the metric as follows:

$$
\omega^{1}=\exp \left(\frac{L_{w}^{2}}{2 \lambda^{2}}\right) d v, \omega^{2}=0, \omega_{j}^{i}=0, \gamma=\eta_{r e f},
$$

where $v$ and $w$ are the coordinates with respect to the orthonormal frame field to the isophotes and substituting these choices into equations (4), (6) and (7). The dynamic scale-spaces of the input image are then governed by the following Cauchy problem:

$$
\frac{\partial L}{\partial s}=\exp \left(-\frac{L_{w}^{2}}{\lambda^{2}}\right) \frac{\partial^{2} L}{\partial v^{2}}, L(\cdot, 0)=L_{0}(\cdot),
$$

which is just an alternative to the controlled Euclidean shortening flow considered in e.g. [2].

\subsection{Image Formation}

In anisotropic scale-space theories [2] one is concerned in retaining the discontinuity sets as much as possible under the smoothing. It is demonstrated in [1] that these theories have a nice geometric foundation. Instead of smoothing the input image by means of the inhomogeneous group actions as proposed in the first reference in the sequel the smoothing of the image formation is presented as proposed in [1]. Because the image formation of a grey-valued input image on a two-dimensional Euclidean space $E^{2}$ can be described as an inhomogeneous Euclidean group action induced by the input image it is natural to diffuse the translation vector field and the rotation vector fields (see section 2). As noted in geometric formulations of the anisotropic scale-space theories [1] the smoothing of the input image can be suppressed at discontinuity sets, such as ridges and ruts. Analogously, the smoothing of the image formation can be suppressed at such objects. Leaving them as much as possible untouched the relaxation of the translation vector field $b$ and the rotation vector fields $f_{i}$ can be defined as follows.

Definition 8. A dynamic scale-space theory for the translation vector field $b$ and the Frank vector fields $f_{i}$ is governed by the following Cauchy problem:

$$
\begin{aligned}
\frac{\partial \psi}{\partial \tau} & =\gamma^{i j} \nabla_{i}\left(O_{\psi} \psi\right)_{j}, \text { on } \Omega \times \mathbb{R}^{+} \\
\psi(x, 0) & =\psi_{0}(x), \text { on } \Omega \\
\gamma^{i j}\left(O_{\psi} \psi\right)_{j} n_{j} & =0, \text { on } \partial \Omega \times \mathbb{R}^{+}
\end{aligned}
$$

with $\psi$ the translation vector field or the matrix of rotation vector fields, $O_{\psi}$ a diffusion operator consistent with $\psi$, and the induced (not necessarily) metric connection $(\Gamma, \gamma)$ such that the components of the metric tensor are given by $\gamma_{i j}=\gamma_{r e f}\left(\hat{\psi}_{i}, \hat{\psi}_{j}\right)$ in which $\hat{\psi}_{i}$ are so-called canonical fields. 
Note that in the case of smoothing of the translation vector field $b$ the metric becomes degenerate. Furthermore, in analogy with the anisotropic scale-space theories [2] the flow $j=-O_{\psi} \psi$ can be set equal to:

$$
j=-d \nabla \psi, d=\exp \left(-\alpha_{\phi} \psi_{\imath} \psi_{j}\right),
$$

with $\alpha_{\phi}$ a contrast parameter. On the basis of the divergence formulation of these dynamic scale-space theories the evolution of the image formation in terms of the translation vector field and the rotation vector field can readily be computed [2]. Concluding these dynamic scale-space theories enable a multi-scale description of the image formation in terms of scaled measures of torsion and curvature. Note that similar dynamic scale-space theories can be formulated, if the image formation should be invariant under the group of monotonic grey-value transformations.

\section{Discussion}

Modern geometry is demonstrated to be useful in describing the image formation of a grey-valued input image. Furthermore, it enables us to come up with dynamic scale-space theories in particular for the image formation.

The acquired geometric expertise allows us to conceive an image as a finite CW-complex in which the ridges, ruts and other type of singularity sets are the essential physical objects bordering different image formation processes. Subsequently, the topological aspects of image induced paths on such a CW-complex can be quantified in terms of so-called (generalised) Vassiliev invariants characterising dynamical processes involved in the image formation.

In the context of dynamic scale-space theories it might be ultimately interesting to formulate also theories that are topologically equivalent or, as physicists say, that are covariant. According to this author one then has to turn to theories taking the landscape of ridges and ruts, and the total grey-values in between as a finite $\mathrm{CW}$-complex. The total grey-values attained at the singularities can savely be redistributed according to the valencies and the couplings on the CWcomplex. One might conjecture that, depending on the particular paradigm chosen to achieve a task certain types of dynamical processes will survive, whereas others definitively will fade out. The outcomes of further research in this area might have some considerable impact in autonomous system research, cognitive sciences and the field of artificial intelligence.

\section{References}

1. Salden, A.H.: Dynamic Scale-Space Paradigms. PhD thesis, Utrecht University, The Netherlands, November, 1996, http://www.ceremade.dauphine.fr/ cohen/MSPCV/pgm961128.html.

2. Weickert, J.: Anisotropic Diffusion in Image Processing. PhD thesis, Dept. of Mathematics, University of Kaiserslautern, Germany, January, 1996. 